\title{
An economic evaluation of management strategies to mitigate the negative effect of twinning in dairy herds
}

\author{
R. Mur-Novales, ${ }^{\star} †$ F. Lopez-Gatius, $\neq \S$ P. M. Fricke, ${ }^{*}$ and V. E. Cabrera ${ }^{* 1}$ \\ ${ }^{*}$ Department of Dairy Science, University of Wisconsin-Madison, Madison 53705 \\ †Department of Animal Science, and \\ $\ddagger$ Agrotecnio Center, University of Lleida, Lleida, Spain, 25198 \\ §2Transfer in Bovine Reproduction SLu, Barbastro, Spain, 22300
}

\begin{abstract}
Our objectives were to develop an economic model to estimate the economic impact of twinning in dairy cows and to evaluate management strategies to mitigate the negative economic impact of twinning in dairy herds. A probabilistic tree considering spontaneous embryo reduction, early pregnancy loss, abortion, metritis, retained placenta, and culling rate at $120 \mathrm{~d}$ of the second, at the end of the second, and at the end of the third lactation was developed for a single pregnancy; we also developed 3 management options upon diagnosis of a twin pregnancy: (1) do nothing, (2) induce abortion using $\mathrm{PGF}_{2 \alpha}$, or (3) attempt manual embryo reduction. A value was given to each branch of the tree by simulating cow states on a farm for 1,400 d to encompass 4 consecutive lactations. The incomes considered in the simulations included milk income over feed cost, income from calves, and slaughter value upon culling. The expenses taken into account depending on each branch included additional inseminations and synchronization protocols, embryo reduction, induction of abortion, replacement heifers, and costs due to metritis and retained placenta. The gross value for a singleton pregnancy and the 3 management options upon diagnosis of a twin pregnancy were calculated by adding the value of all braches multiplied by their probability. The costs for the 3 management options were calculated by subtracting its gross value minus the gross value of a singleton pregnancy. The negative economic impact of a twin pregnancy ranged from $\$ 97$ to $\$ 225$ depending on the type of twin pregnancy (unilateral vs. bilateral), parity, and DIM when the twin pregnancy occurred. The overall negative economic impact of twinning on dairy farm profitability in the United States was estimated to be $\$ 96$ million per year. Attempting manual
\end{abstract}

Received January 5, 2018.

Accepted May 9, 2018

${ }^{1}$ Corresponding author: vcabrera@wisc.edu embryo reduction early during gestation upon diagnosis of a twin pregnancy was the optimal management strategy for mitigating the negative economic impact of twinning under a wide variety of scenarios.

Key words: economic modeling, reproductive management

\section{INTRODUCTION}

Cattle are monotocous, meaning that, under most circumstances, a successful pregnancy results in the birth of a single calf. Occasionally, however, the reproductive process in cattle, as with many other monotocous species, results in the birth of twins. Twinning in dairy cows is undesirable because it decreases the overall profitability of a dairy farm through combined negative effects on cows calving twins as well as on calves born as twins (Fricke, 2001). Cows calving twins are at greater risk for many reproductive disorders, including retained placenta, dystocia, and metritis, as well as metabolic disorders, including displaced abomasum and ketosis (Markusfeld, 1987; Nielen et al., 1989), all of which increase the risk of culling (Eddy et al., 1991). The incidence of abortion, stillbirth, neonatal calf mortality, and reduced birth weight are greater among calves born as twins than singletons, probably due to reduced gestation length and increased incidence of dystocia among cows calving twins (Nielen et al., 1989). Taken together, these negative effects on reproduction, periparturient disorders, and calf health have a negative economic impact on dairy farm profitability (Eddy et al., 1991; Beerepoot et al., 1992).

Reported rates of twinning in dairy herds have increased over time, concurrent with increases in milk production (Kinsel et al., 1998; Silva del Rio et al., 2007), and this trend for increased twinning over time will likely continue into the future (López-Gatius et al., 2017). Several studies have been undertaken to mitigate or prevent the negative effect of twinning (Fricke, 2015; López-Gatius et al., 2017). Potential strategies include genetic selection against twinning (Johanson et 
al., 2001), increasing circulating progesterone during a synchronized follicular wave to decrease the incidence of double ovulations and dizygous twinning through the use of hormonal synchronization protocols (Wiltbank et al., 2000; Fricke, 2015), or differential dry period feeding strategies for cows carrying twin pregnancies during late gestation (Silva del Río et al., 2010).

From a clinical perspective, twin pregnancies can accurately be diagnosed by d 30 of gestation using transrectal ultrasonography (López-Gatius and García-Ispierto, 2010; López-Gatius and Hunter, 2017). Pregnancy loss during the early fetal period (d 28-90 of gestation) for cows carrying twins is 3 to 7 times greater than for cows with a singleton pregnancy and 5 to 9 times greater for unilateral than for bilateral twins (Silva del Río et al., 2009; López-Gatius et al., 2017). Upon diagnosis of twins, veterinarians can decide to do nothing or to induce abortion using $\mathrm{PGF}_{2 \alpha}$ to avoid problems associated with calving twins despite the economic losses associated with early pregnancy loss (Cabrera, 2014). Natural or spontaneous embryo reduction from an initial diagnosis of twins for cows that continue gestation ranges from 11 to $28 \%$ (Silva del Río et al., 2009; López-Gatius and Hunter, 2017; López-Gatius et al., 2017). Because the presence of a dead co-twin is detected in some of these cows at pregnancy diagnosis, the best time for an embryo reduction approach should be immediately after confirming that both twins are alive based on transrectal ultrasonography.

Because the rate of twinning can exceed $18 \%$ in some herds (Silva del Rio et al., 2007; Andreu-Vázquez et al., 2012a), a method for induced embryo reduction has been developed and assessed. Manual rupture of the amniotic vesicle or transvaginal ultrasound-guided aspiration of allanto-amniotic fluid or intraluteal instillation of $\mathrm{PGF}_{2 \alpha}$ in 1 of the 2 corpora lutea have been proposed as methods to decrease twinning in dairy cows from d 28 to 41 of gestation (López-Gatius, 2005; Andreu-Vázquez et al., 2011, 2012b; López-Gatius and Hunter, 2016). Manual embryo reduction aims to eliminate 1 embryo by manually crushing the amniotic vesicle of 1 of the embryonic vesicles without damaging its co-twin during early gestation, when most spontaneous embryo reduction events occur (Silva del Río et al., 2009; López-Gatius and Hunter, 2017). An embryo reduction is considered successful when the remaining co-twin becomes a viable single pregnancy. Success rates up to $71.4 \%$ in bilateral and $46.2 \%$ in unilateral twin pregnancies have been reported for manual embryo reduction (Andreu-Vázquez et al., 2011).

The objective of the present study was to develop an economic model to estimate the negative economic impact of twinning in dairy herds and to evaluate 3 management strategies upon diagnosis of a twin preg- nancy in a commercial dairy herd. The 3 management options modeled included (1) do nothing (DN), (2) induction of abortion using $\mathrm{PGF}_{2 \alpha}(\mathbf{P G})$, and (3) attempt manual embryo reduction (ER).

\section{MATERIALS AND METHODS}

\section{Overview of the Economic Model}

An economic model was developed in Microsoft Excel (Microsoft Corp., Redmond, WA) to evaluate 3 management options upon diagnosis of a twin pregnancy early during gestation. Options evaluated were (1) do nothing, (2) induce abortion by treating with $\mathrm{PGF}_{2 \alpha}$, or (3) attempt manual embryo reduction. A probabilistic tree considering spontaneous embryo reduction, early pregnancy loss, abortion, metritis, retained placenta, and culling rate at $120 \mathrm{~d}$ of the second, at the end of the second, and at the end of the third lactation was developed for a singleton pregnancy and the 3 management options for a twin pregnancy (Figure 1). Probabilities were derived from the scientific literature and expert knowledge from the field (Table 1). A value was given to each branch of the probabilistic tree by simulating a cow state (CS) on a farm for 1,400 consecutive days to encompass the time required for a cow to complete four 305-d lactations assuming a 60-d dry period. The gross value of every management option $\left(\mathrm{GV}_{\mathrm{i}}\right)$ was calculated as

$$
\mathrm{GV}_{\mathrm{i}}=\Sigma \mathrm{p}_{\mathrm{i}} \times \mathrm{V}_{\mathrm{i}},
$$

where $\mathrm{p}_{\mathrm{i}}=$ probability of every branch and $\mathrm{V}_{\mathrm{i}}=$ value of every branch. Finally, the net cost of different management strategies was the result of subtracting the value of a single pregnancy from the value of the 3 potential management decisions for a twin pregnancy.

\section{Calculation of Branch Value}

The incomes considered in the simulation model included milk income over feed cost (IOFC), income from calves born, and slaughter value when culling occurred. The extra expenses taken into account depending on each branch included additional inseminations and synchronization protocols, embryo reduction, induction of abortion, replacement heifers, and costs due to metritis and retained placenta.

\section{Calculation of Milk IOFC}

The IOFC for each case was calculated by subtracting the feed costs from the milk value produced during the 1,400-d simulation period. 


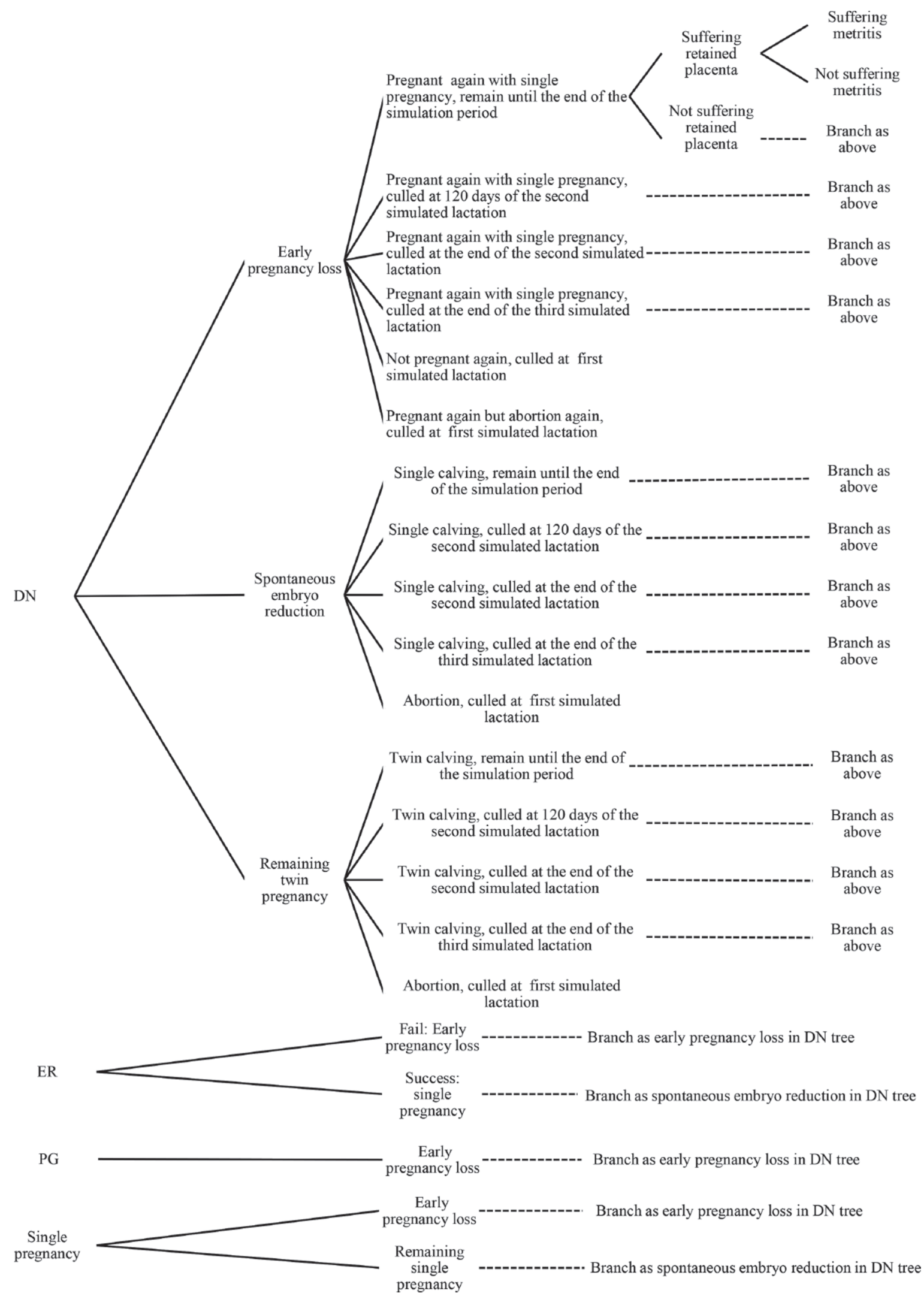

Figure 1. Probabilistic trees showing all outcomes for a singleton pregnancy and the 3 management options [do nothing (DN), embryo reduction (ER), or induce abortion (PG)] and their outcomes for a twin pregnancy. 
Lactation Lengths. The first step to calculate the IOFC was to determine the length of all lactations included in the simulation. Because the aim of our study was to compare 3 management options after diagnosing a twin pregnancy, the twin pregnancy always occurred within the first 240-d period that was simulated, and the consequences of the twin pregnancy were modeled for 1,400 d from the beginning of the lactation when the twin pregnancy occurred. Thus, the length of the first lactation simulated $\left(\mathrm{DIM}_{1}\right)$ varied in every case and was calculated using the following formula:

$$
\mathrm{DIM}_{1}=\mathrm{CCI}_{\mathrm{S}}+\mathrm{DPL}+\mathrm{DPA}+\mathrm{GL}-\mathrm{DRY}
$$

where $\mathrm{CCI}_{\mathrm{S}}=$ simulated calving to conception interval, $\mathrm{DPL}=$ days of gestation when early pregnancy loss or abortion occurs, DPA $=$ days required to re-establish pregnancy, GL = gestation length, and DRY = dry period duration. We assumed that after a pregnancy loss all cows were enrolled in an 8-d synchronization protocol that included a progesterone insert to allow for timed AI (Santos et al., 2016). Cows that failed to become pregnant to timed AI after this protocol had the chance to be reinseminated every $21 \mathrm{~d}$ thereafter. Replacement occurred when cows failed to become pregnant by the time of a predefined DIM cutoff for AI services $\left(\mathbf{D I M}_{\text {cutoff }}\right)$ and they reached a minimum milk threshold (Giordano et al., 2012). Therefore, if $\mathrm{CCI}_{\mathrm{S}}+\mathrm{DPL}+\mathrm{DPA}>\mathrm{DIM}_{\text {cutoff }}$, the length of the first lactation $\left(\mathrm{DIM}_{1}\right)$ was the day when milk production was below a milk threshold ( $\left.\mathrm{DIM}_{\text {milk threshold }}\right)$, or $\mathrm{CCI}_{\mathrm{S}}+$ DPL if $\mathrm{CCI}_{\mathrm{S}}+\mathrm{DPL}>\mathrm{DIM}_{\text {milk threshold }}$.

Because calving twins affects reproductive performance during the subsequent lactation, the length of the second lactation $\left(\mathrm{DIM}_{2}\right)$ was calculated as

$$
\mathrm{DIM}_{2}=\mathrm{CCI}_{\text {farm }}+\mathrm{CCI}_{\text {twins }}+\mathrm{GL}-\mathrm{DRY}
$$

where $\mathrm{CCI}_{\text {farm }}=$ average calving to conception interval of the farm and $\mathrm{CCI}_{\text {twins }}=$ calving to conception interval increase after a twin calving. The $\mathrm{CCI}_{\text {twins }}$ applied in the simulations was $26 \mathrm{~d}$ (Andreu-Vázquez et al., 2012a). In some cases (Figure 1), culling was forced to occur at $120 \mathrm{~d}$ of the second simulated lactation

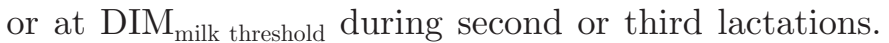
As shown below, the length of the remaining lactations and the replacement lactations $\mathrm{DIM}_{\mathrm{i}}$ were considered as farm average lactations until a cow reached the end of the simulation period at 1,400 d:

$$
\mathrm{DIM}_{\mathrm{i}}=\mathrm{CCI}_{\mathrm{farm}}+\mathrm{GL}-\mathrm{DRY} .
$$

The values considered in each case for DPL, DPA, $\mathrm{DIM}_{\text {cutoff }}, \mathrm{CCI}_{\text {farm }}$, and the milk threshold are shown in

Table 1. Probabilities applied in the probabilistic trees to calculate the net cost of the 3 management options after the diagnosis of a twin pregnancy [do nothing (DN), embryo reduction (ER), or induce abortion (PG)] and the breakeven point of ER

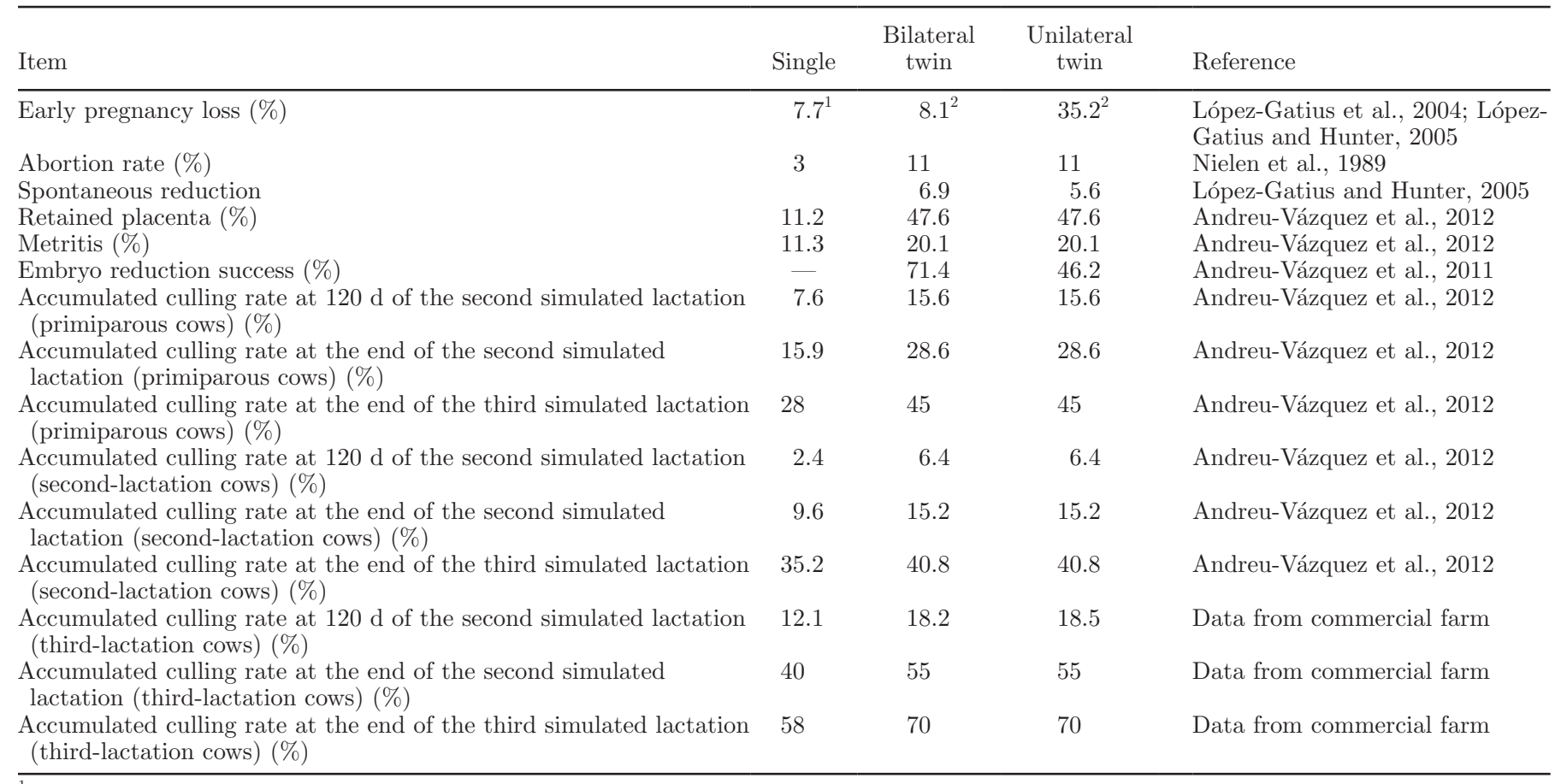

${ }^{1}$ Value from Lopez-Gatius et al., 2004.

${ }^{2}$ Value from Lopez-Gatius and Hunter, 2005. 
Table 2. Values used to calculate gestation length and the net cost of the 3 management options after the diagnosis of a twin pregnancy [do nothing (DN), embryo reduction (ER), or induce abortion (PG)] and the breakeven point of ER

\begin{tabular}{lccccc}
\hline Item & Single & $\begin{array}{c}\text { Do } \\
\text { nothing }\end{array}$ & $\begin{array}{c}\text { Embryo } \\
\text { reduction }\end{array}$ & $\begin{array}{c}\text { Induce } \\
\text { abortion }\end{array}$ & Reference \\
\hline Moment of early pregnancy loss (d after AI) & $52^{1}$ & $75^{1}$ & $42^{2}$ & $30^{2}$ & $\begin{array}{l}\text { López-Gatius et al., 2004; } \\
\text { extended farm routine }\end{array}$ \\
Moment of abortion (d after AI) & 185 & 185 & 185 & 185 & $\begin{array}{l}\text { Data from commercial farm } \\
\text { Data from commercial farm }\end{array}$ \\
\hline Farms average calving to conception interval (d) & 120 & 120 & 120 & 120 & com
\end{tabular}

${ }^{1}$ Value from Lopez-Gatius et., 2004.

${ }^{2}$ Embryo reduction and abortion induction carried out immediately after pregnancy diagnosis (30 d). Pregnancy losses occasioned by unsuccessful embryo reduction were found at pregnancy confirmation $(42 \mathrm{~d})$. Cows were re-enrolled in synchronization protocols right after the induced abortion or when the pregnancy was found to be lost after embryo reduction.

Tables 2 and 3. To study the effect of twin pregnancies conceived at different times during gestation, the $\mathrm{CCI}_{\mathrm{S}}$ ranged between $60 \mathrm{~d}$ and $\mathrm{DIM}_{\text {cutoff }}$. Because the aim of this simulation was to study the effect of twin pregnancies, the calving to conception interval of all single simulated lactations not affected by a previous twin calving was set as $\mathrm{CCI}_{\text {farm }}$. The values applied for GL and DRY were 280 and 60 for single pregnancies and 274 and 70 for twin pregnancies, respectively (Nielen et al., 1989).

Milk Production. The MilkBot model (Ehrlich, 2011) was used to predict milk production during each lactation. The MilkBot model predicts milk production based on 4 parameters: (a) scale, (b) ramp, $(c)$ offset, and $(d)$ decay (for more details, please refer to http:// milkbot.com and Ehrlich, 2011).

$$
\left[\mathrm{MP}_{\mathrm{DIM}}=a \times\left(1-\frac{e^{\left(\frac{c-\text { DIM }}{b}\right)}}{2}\right) \times e^{(-d \times \mathrm{DIM})}\right]
$$

The total amount of milk produced in each simulated lactation was calculated by integrating the previous function. The parameters used in the MilkBot model (Table 4) were obtained by fitting lactation benchmark curves for Wisconsin dairy farms provided by AgSource Cooperative Services (AgSource.crinet.com; Verona, WI) using the milk curve fitter tool from the University of Wisconsin-Madison Dairy Management website (http://dairymgt.info/tools/mcf_online/newindex .php). We assumed that lactation curve parameters were similar for cows in their third and later lactations.

DMI. The DMI (kg/cow per day) was a function of maintenance and milk production according to BW $(\mathrm{kg})$ and $4 \%$ FCM being produced, calculated according to NRC (2001):

$$
\begin{aligned}
\mathrm{DMI}_{\mathrm{DIM}} & =\left(0.372 \times \mathrm{FCM}_{\text {DIM }}+0.0968 \times \mathrm{BW}^{0.75}\right) \times \\
& \left(1-e^{0.192 \times\left(\frac{\mathrm{DIM}}{7}\right)+3.67}\right)
\end{aligned}
$$

$\boldsymbol{B} \boldsymbol{W}$. The BW was calculated for each cow using the Korver function as described by van Arendonk (1985) as a cumulative function of the age of a cow (according to parity), DIM, and pregnancy status, using a similar approach as reported by Kalantari et al. (2016). Simulated cows were assumed to be Holstein and entered the simulation at 0 DIM with $540 \mathrm{~kg}$ of BW for primiparous cows and $620 \mathrm{~kg}$ of BW for multiparous cows.

\section{Calf Value Calculations}

Calf value calculations were based on data from Silva del Río et al. (2007), in which a single calving produced 0.93 live calves (53.3\% males and $46.7 \%$ females), whereas a twin calving produced 1.7 live calves $(51.9 \%$ males, $21.8 \%$ females from a male-female gestation

Table 3. Values used to calculate days to pregnancy after a pregnancy loss (DPA) and the moment of culling when a nonpregnant cow reaches a milk threshold $\left(\mathrm{DIM}_{\text {milk threshold }}\right)$ in the simulations to calculate the net cost of the 3 management options after the diagnosis of a twin pregnancy [do nothing (DN), embryo reduction (ER), or induce abortion $(\mathrm{PG})]$ and the breakeven point of ER

\begin{tabular}{lr}
\hline Parameter & Value \\
\hline Conception rate for first resynchronization after pregnancy loss (\%) & 35 \\
21-d pregnancy rate after resynchronization after pregnancy loss (\%) & 25 \\
DIM cutoff for AI services (d) & 250 \\
Minimum milk threshold (kg) & 25 \\
\hline
\end{tabular}


Table 4. Parameters used in the MilkBot model (Ehrlich, 2011) to calculate the milk production in the simulations to calculate the net cost of the 3 management options after the diagnosis of a twin pregnancy [do nothing $(\mathrm{DN})$, embryo reduction (ER), or induce abortion $(\mathrm{PG})]$ and the breakeven point of ER

\begin{tabular}{lcccrc}
\hline $\begin{array}{l}\text { Average farm milk production } \\
\text { (kg of milk/cow per year) }\end{array}$ & Lactation & Scale & Ramp & Offset & Decay \\
\hline 9,979 & 1 & 36.37 & 24.91 & -2.19 & 0.0011 \\
& 2 & 45.80 & 15.44 & -1.15 & 0.0019 \\
& $>2$ & 49.06 & 18.43 & -0.31 & 0.0021 \\
& 1 & 37.29 & 25.57 & -1.53 & 0.0010 \\
& 2 & 47.50 & 16.86 & -1.17 & 0.0019 \\
& $>2$ & 51.03 & 18.60 & 0.45 & 0.0021 \\
\hline
\end{tabular}

and $26.3 \%$ females from a female-female gestation). The value assigned to female calves born from malefemale gestations was set at $70 \%$ of a male calf value, to account for females expected to be freemartins (i.e., not usable as replacements) and have reduced ability to produce beef compared with male calves (Padula, 2005).

\section{Culling Value Calculations}

The culling value was calculated by multiplying the BW at culling by the market price of a kilogram of a live cow.

\section{Additional Reproductive Cost Calculation}

Synchronization Protocols. In all cases when early pregnancy loss or abortion occurred before DIM $_{\text {cutoff }}$, the cost of an 8-d progesterone protocol for timed AI was added. The calculation included $5 \mathrm{~min}$ of reproductive specialist labor for administration of hormonal treatments and the cost of the hormones.

$\boldsymbol{A} \boldsymbol{I}$. Only in the branches where pregnancy loss occurred before DIM $_{\text {cutoff }}$ or when calving to conception interval increased due to a twin calving were extra inseminations included. After a pregnancy loss, the number of extra inseminations was calculated by dividing by 21 the number of days since the end of the protocol until a new conception or until DIM cutoff $_{\text {The }}$ duration of the timed AI protocol was then added. In cases where calving to conception interval increased as a consequence of a twin calving, the number of extraordinary inseminations was calculated by diving $\mathrm{CCI}_{\text {twins }}$ by 21 . The cost of an insemination included semen and labor to perform the insemination.

Embryo Reduction. The cost of an embryo reduction included 5 min of reproductive specialist labor, the cost of 1 dose of flunixin meglumine, and the cost of 1 dose of GnRH.

Induction of Abortion. The cost of induction of abortion included 1 min of reproductive specialist labor and the cost of 1 dose of $\mathrm{PGF}_{2 \alpha}$.

Metritis and Retained Placenta. The cost of metritis and retained placenta included veterinary and treatment costs, milk discarded as a consequence of antibiotic treatment, and a reduction in milk production. The economic losses associated with decreased reproductive performance and increased culling as a result of metritis and retained placenta were not included because these costs are accounted for in other parts of the model. When a cow suffered both pathologies, the cost included the whole cost of metritis and the veterinary and treatment costs of retained placenta. This calculation and the values applied to it were adapted from Liang et al. (2017; Table 5).

\section{Replacement Cost}

The replacement cost included only the market value of a heifer. We assumed that the replacement heifer initiated her first lactation the day after the cow was culled.

Table 5. Values used to estimate the cost of metritis and retained placenta in the simulations of the net cost of the 3 management options after the diagnosis of a twin pregnancy [do nothing (DN), embryo reduction (ER), or induce abortion $(\mathrm{PG})]$ and the breakeven point of $\mathrm{ER}^{1}$

\begin{tabular}{lccc}
\hline Disease & $\begin{array}{c}\text { Veterinary, labor and } \\
\text { treatment cost }(\$ / \text { cow })\end{array}$ & $\begin{array}{c}\text { Decreased milk } \\
\text { production }(\mathrm{kg} / \mathrm{cow})\end{array}$ & $\begin{array}{c}\text { Milk discarded } \\
(\mathrm{kg} / \mathrm{cow})\end{array}$ \\
\hline Metritis & 100 & 24 & 110 \\
Retained placenta & 84 & 240 & $\mathrm{NA}^{2}$ \\
\hline
\end{tabular}

${ }^{1}$ Adapted from Liang et al. (2017).

${ }^{2} \mathrm{NA}=$ not applicable. 
Table 6. Costs used in the simulations to calculate the net cost of the 3 management options after the diagnosis of a twin pregnancy [do nothing (DN), embryo reduction (ER), or induce abortion (PG)] and the breakeven point of ER

\begin{tabular}{llcl}
\hline Item & Unit & Price & Reference \\
\hline Milk & $\$ / \mathrm{kg}$ & 0.36 & Giordano et al., 2012 \\
Lactation diet & $\$ / \mathrm{kg}$ & 0.17 & Giordano et al., 2012 \\
Dry diet & $\$ / \mathrm{kg}$ & 0.13 & Giordano et al., 2012 \\
Male calf & $\$ /$ head & 50 & Giordano et al., 2012 \\
Female calf & $\$ /$ head & 136 & Giordano et al., 2012 \\
Culling & $\$ / \mathrm{kg}$ of a live cow & 1.16 & Giordano et al., 2012 \\
Heifer & $\$ /$ head & 1,302 & Giordano et al., 2012 \\
Dose of $\mathrm{PGF}_{2 \alpha}$ & $\$ /$ dose & 2.3 & Giordano et al., 2012 \\
Dose of $\mathrm{GnRH}$ & $\$ /$ dose & 2.6 & Giordano et al., 2012 \\
Progesterone device & $\$ /$ dose & 12 & Data from commercial farm \\
AI & $\$ /$ AI & 10 & Giordano et al., 2012 \\
Reproductive specialist labor & $\$ /$ h & 105 & Giordano et al., 2012 \\
Dose of flunixin meglumine & $\$ /$ dose & 14 & Pfeifer et al., 2008 \\
\hline
\end{tabular}

${ }^{1}$ Includes semen and labor costs.

\section{Costs Used in the Simulations}

All costs not specified above that were used in the simulations are shown in Table 6. Costs used in the simulations were collected from the scientific literature published within the last $10 \mathrm{yr}$ as well as actual costs incurred by commercial dairy farms.

\section{Simulations}

Cost of a Twin Pregnancy. To cover as many as possible situations as would occur on a commercial dairy farm, bilateral and unilateral twin pregnancies occurring from the end of the voluntary waiting period $(60 \mathrm{DIM})$ to the $\mathrm{DIM}_{\text {cutoff }}(250 \mathrm{DIM})$ by first-, second-, or third-lactation cows were simulated. First, a comparison between single pregnancies and twin pregnancies was made after applying the DN management option to determine the net cost of a twin pregnancy. Then, to evaluate the different management options after diagnosing a twin pregnancy, the net cost after attempting ER or PG were calculated and compared with the DN management option.

Breakeven Analysis for Embryo Reduction Success Rate. Because ER net cost was directly related to its success rate, a breakeven analysis was performed for this parameter. The breakeven point occurs when the economic benefit or net value of one management option over another becomes zero. The embryo reduction success rate was then changed until the net cost of ER was equal to the lowest net cost between the DN and PG management options for every simulated scenario.

Sensitivity Analysis. Sensitivity analyses were used to determine the effect of changing a specific parameter while keeping the others constant on the net cost of twin pregnancies after the 3 different management options.
Parameters such as average herd milk production, relative individual cow milk production within a herd, milk price, fertility after pregnancy loss, or days in gestation when pregnancy loss occurred were evaluated (Table 7). Net cost for all management options and the profit of the most economical management alternative when compared with the DN management option were calculated across all scenarios.

\section{RESULTS AND DISCUSSION}

\section{Cost of a Twin Pregnancy}

The cost of a twin pregnancy when no management action is taken (i.e., DN) depended on the type of twin pregnancy (unilateral vs. bilateral), parity, and DIM when the twin pregnancy was conceived (Figure 2). Unilateral twin pregnancies had a greater cost during early and mid lactation than bilateral twin pregnancies, but when lactation approached the DIM $_{\text {cutoff }}$, set at 250 DIM for all simulations, unilateral twin pregnancies became less costly than bilateral twin pregnancies. Differences due to twin pregnancy type occurred because of the greater risk of early pregnancy loss for unilateral twin pregnancies than for bilateral twin pregnancies. López-Gatius and Hunter (2005) reported 35.2 and $8.1 \%$ as the risk of early pregnancy loss for unilateral and bilateral twin gestations, respectively.

Both early pregnancy loss and extended lactations have a high negative economic impact on dairy herds (Cabrera, 2014). When twin pregnancies occurred late during the breeding period (Figure 3), a greater number of cows failed to conceive due to the increased risk of culling before the DIM cutoff for insemination after suffering a pregnancy loss. This increased culling decreased the number of cows completing long lactations 


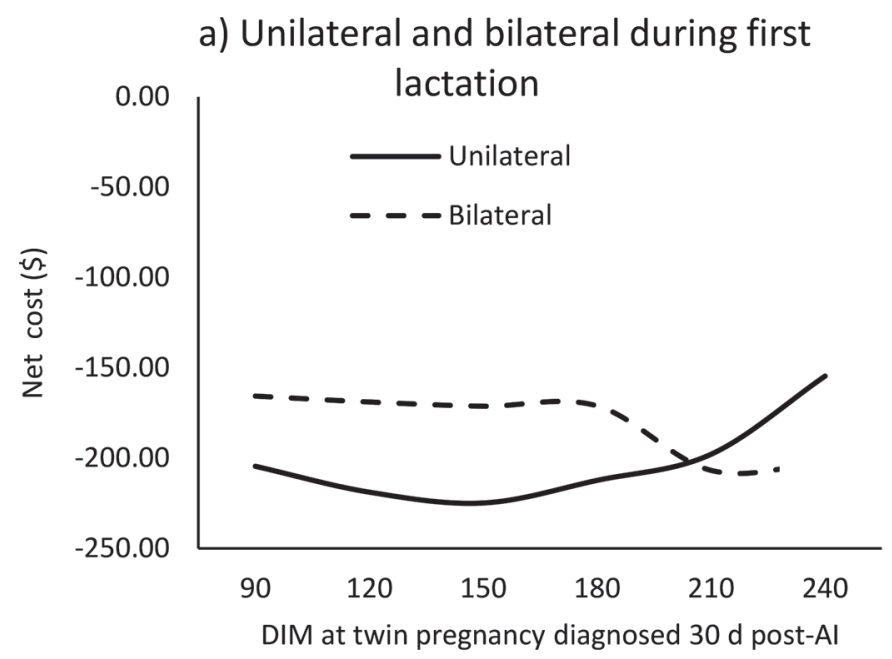

b) Unilateral

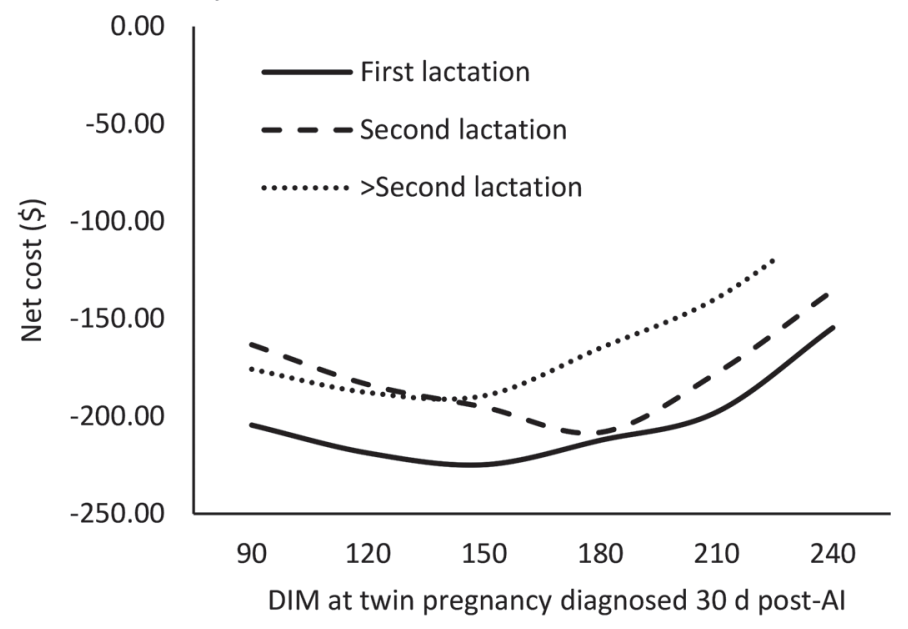

c) Bilateral

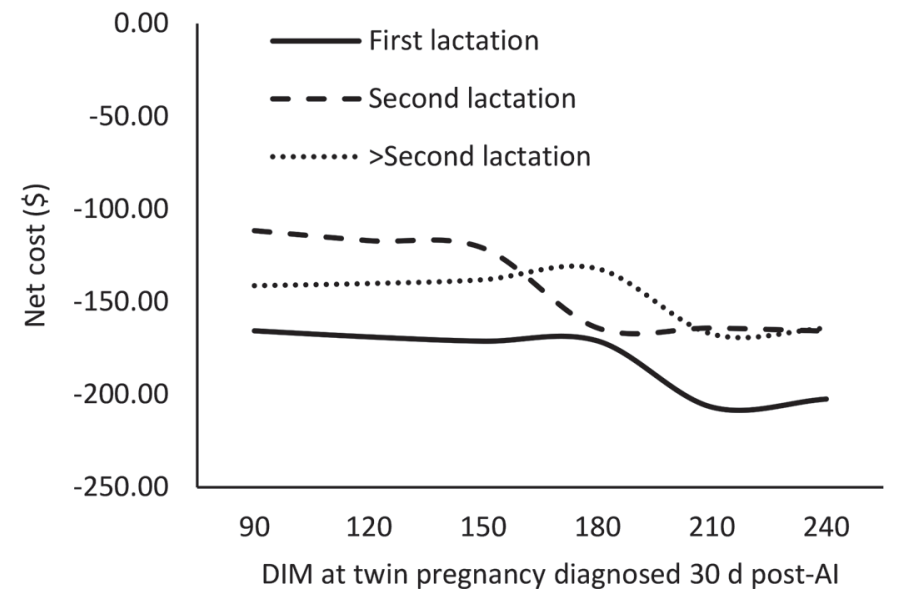

Figure 2. Net cost of a twin pregnancy compared with a singleton pregnancy conceived at the same DIM for a bilateral and a unilateral first lactation twin pregnancy (a), unilateral twin pregnancy for different lactations (b), and bilateral twin pregnancy for different lactations (c).

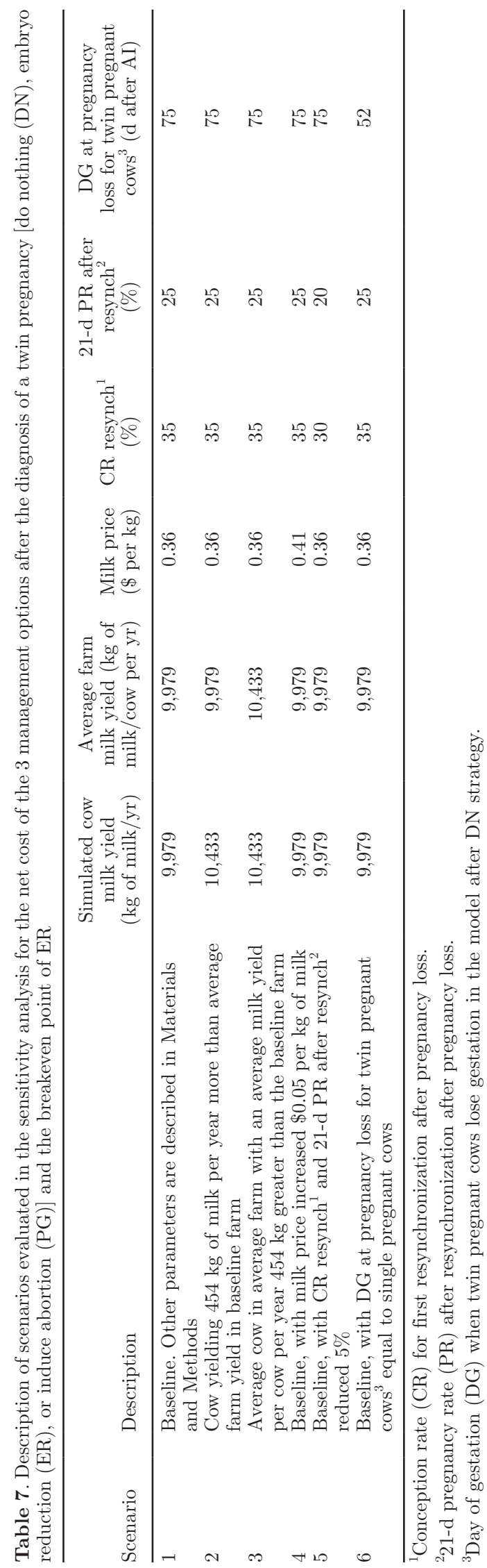


after the minimum milk production threshold. Thus, when cows conceived twins late during the breeding period, the negative economic impact associated with a higher risk of early pregnancy loss and the subsequent replacement cost of unilateral twin pregnancies was compensated for by the decreased number of cows completing long extended lactations before suffering all of the negative consequences associated with a twin calving. This phenomenon was more marked for multiparous than for primiparous cows (Figure 2), because primiparous cows have more persistent lactation curves than multiparous cows (Ehrlich, 2011).

Twin pregnancies had a greater negative effect for primiparous than for multiparous cows (Figure 2) because multiparous cows produce a greater IOFC than primiparous cows (Cabrera, 2012), and a twin calving increases the risk of early culling more for primiparous than for multiparous cows (Andreu-Vázquez et al. 2012a). As a consequence of the greater IOFC of multiparous cows, profit increases as the proportion of multiparous cows increased. If a given cow state was initially for a primiparous cow that was culled and replaced by another primiparous cow during the simulation period, that cow state was never occupied by a cow in the optimal stage of its productive life. As a consequence, that cow state had the lowest value among all simulated branches.

The negative effect of a twin pregnancy ranged from $\$ 97$ to $\$ 225$, and in most cases was greater than the $\$ 96$ calculated by Eddy et al. (1991) and the $\$ 107$ calculated by Beerepoot et al. (1992) for multiparous cows. The difference between reported negative values for a twin pregnancy might be explained because early pregnancy losses were not accounted for in Eddy et al. (1991) and Beerepoot et al. (1992). In their studies, Eddy et al. (1991) and Beerepoot et al. (1992) also applied increased culling rate only during the subsequent lactation of a twin calving. Furthermore, in those evaluations, all calculations were carried out at the cow level instead of using cow states. Finally, milk yields have substantially increased since the time of previous reports. Based on the obtained values, we estimate that a $1 \%$ decrease in twinning rate accomplished either by genetic selection (Johanson et al., 2001) or by increasing progesterone during follicular growth (Fricke, 2015) can represent a profit of $\$ 0.97$ to $\$ 2.25$ per pregnant cow $[\$ 227$ to $\$ 526$ for the average farm size in the United States containing 234 cows (USDA, 2017)]. These values should be taken into account when evaluating reproductive and genetic programs and can be used as cost thresholds when evaluating specific actions to prevent twin gestations. In 2001, the negative economic

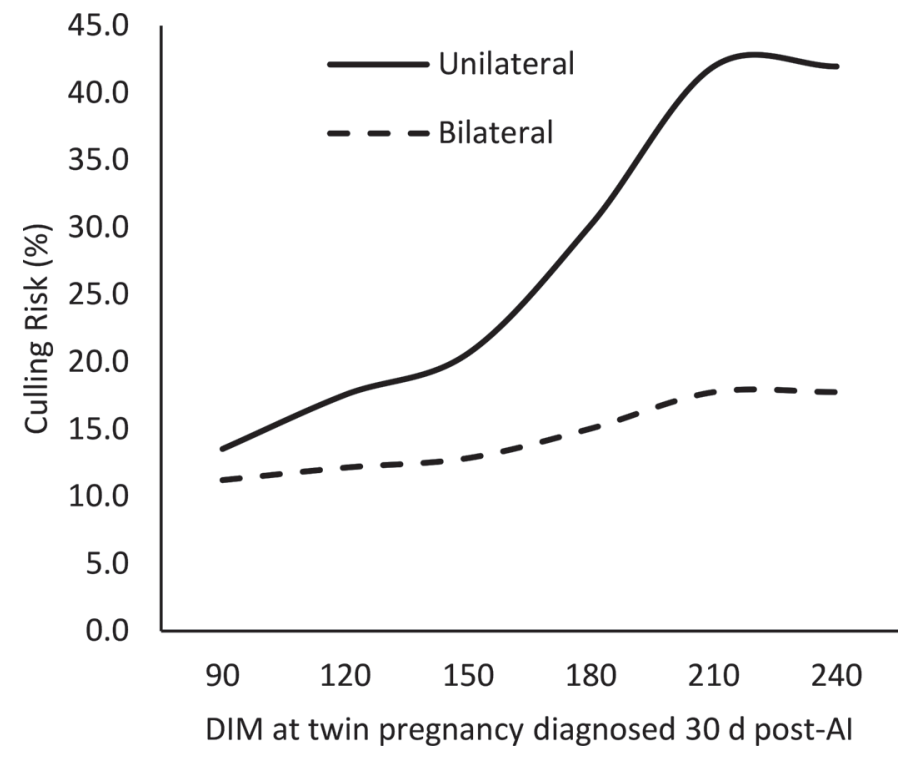

Figure 3. Culling risk due to reproductive reasons after diagnosing a twin pregnancy in the current lactation.

impact of twinning in the United States was estimated to be $\$ 55$ million (Johanson et al., 2001). Extrapolating our average cost for a twin pregnancy (\$161), along with a $4.8 \%$ rate of twin calvings (Silva del Rio et al., 2007) that before early pregnancy loss was $6.4 \%(4.8 / 0.755$ $=6.4$; Silva del Río et al., 2009) of twin pregnancies and the current US inventory of 9.3 million dairy cows (USDA, 2017), we estimate the annual negative effect of twinning in the United States to be $\$ 96$ million. The same calculation for the average farm size in the United States resulted in $\$ 2,415 /$ farm per year.

\section{Management Options After Diagnosing a Twin Pregnancy}

To date, no economic models have been made available to evaluate potential management strategies after diagnosing a twin pregnancy. Because of the reported increase in twinning over time (Kinsel et al., 1998; Silva del Rio et al., 2007), veterinarians are uncertain about the best management decision to make upon diagnosis of a twin pregnancy in the field. Differential feeding strategies during the dry period were not evaluated in our study because increasing the duration of the closeup ration during the dry period was reported not to mitigate the negative effects of twin calvings (Silva del Rio et al., 2010). The net cost pattern throughout lactation for the 3 management options evaluated in our study was similar in all simulated parities (Figure 4). Similar to doing nothing, induction of abortion 

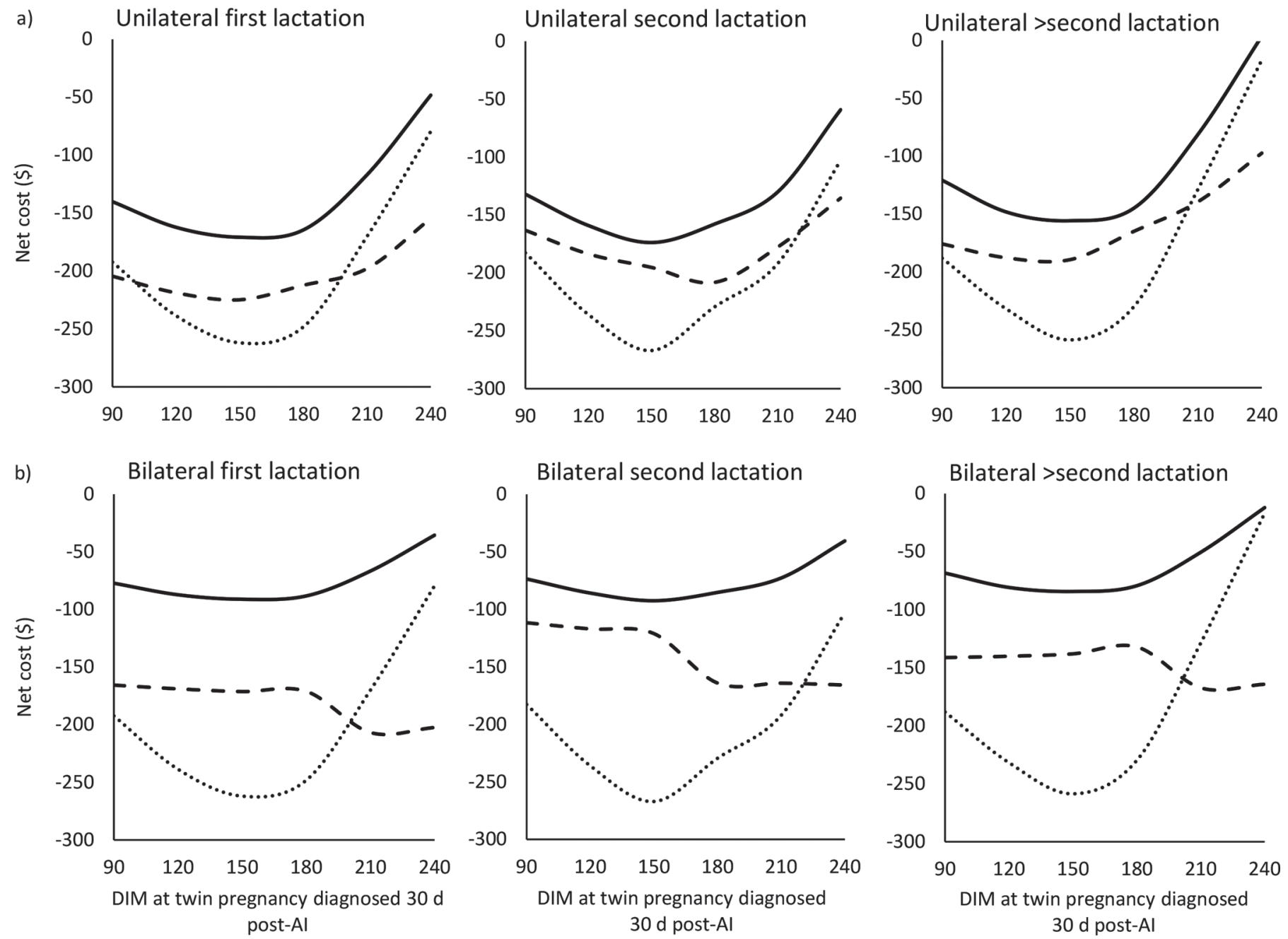

Figure 4. Net cost of a twin pregnancy after the 3 management options compared with a singleton pregnancy conceived at the same DIM for unilateral (a) and bilateral (b) twin pregnancies in different lactations.

and manual embryo reduction also presented different trends for early and mid lactation than when approaching the end of the breeding period.

The option to induce abortion during early or mid lactation was economically the worst alternative because it produced the highest pregnancy loss rate; however, inducing abortion when used close to the DIM limit for insemination improved because it increased the culling rate (Figure 5) and consequently decreased the risk of extended lactations followed by all of the negative effects related to a twin calving.

Overall, attempting manual embryo reduction was economically the best management alternative for any lactation, DIM, or type of twin pregnancy (Figure 4) when the success rate was at least $71.4 \%$ for bilateral and $46.2 \%$ for unilateral twin pregnancies, as reported by Andreu-Vázquez et al. (2011). Even though attempting manual embryo reduction increased pregnancy loss by around $20 \%$ with respect to doing nothing (Table 1), attempting manual embryo reduction had the best economic outcome when attempted during early and mid lactation because it decreased the risk of late abortion and eliminated all negative consequences associated with twin calvings, including increased postpartum disease or decreased lifespan in cows that remained pregnant after a successful embryo reduction. In addition, manual embryo reduction was the best management option at the end of the breeding period because it decreased the probability of late abortion, eliminated the consequences of twin calvings for 
cows that remained pregnant, and decreased the risk of extended lactations (Figure 5).

\section{Breakeven Analysis for Embryo Reduction Success Rate}

Because the cost effectiveness of manual embryo reduction was directly related to its success rate, a breakeven analysis was performed to determine the minimum embryo reduction success rate required to maintain manual embryo reduction as the most economically viable management option upon diagnosis of a twin pregnancy. During early and mid lactation, the economic value for attempting manual embryo reduction was equalized with doing nothing, whereas at the end of the breeding period it was equalized with the economic value of inducing abortion (Figure 4). As a consequence, the breakeven point function (Figure 6) presented differential trends at early and mid lactation than at the end of the breeding period. When manual embryo reduction was equalized to the do nothing alternative, the net cost of a twin pregnancy was greater for the do nothing management option, and the manual embryo reduction success rate needed to achieve the same net cost was decreased (Figures 2 and 6). Despite the observation that manual embryo reduction of unilateral pregnancies was reported to obtain low success rates due to the proximity of the embryos and the anastomosis of their placentas (López-Gatius and
Hunter, 2017), attempting manual embryo reduction remained the most profitable management option. This was because when no action was taken, the probability of pregnancy loss was very high (Table 1). Breakeven points were found at any time of lactation below 40 and $30 \%$ manual embryo reduction success rate for multiparous and primiparous cows, respectively. Thus, in all simulated cases, the breakeven points were lower than the reported success rates for manual embryo reduction (Table 1), reinforcing the fact that this alternative was the most profitable in all simulated situations.

At the end of the breeding period, when manual embryo reduction was equalized to induction of abortion, the breakeven values rapidly dropped to $0 \%$ because inducing an abortion had the same effect as failing all embryo reductions but occurred 2 wk earlier. In the case of inducing abortion resynchronization of all cows began $30 \mathrm{~d}$ after AI, whereas in the case of failing all the embryo reductions resynchronization of all cows began $45 \mathrm{~d}$ after AI.

\section{Sensitivity Analysis}

All simulations carried out for the sensitivity analysis (Table 7) presented the same trends throughout lactation, parity, and type of twin pregnancy similar to those described previously. Thus, only the values calculated for second-lactation cows diagnosed with a bilateral twin pregnancy at 150 DIM are shown in Figure 7.

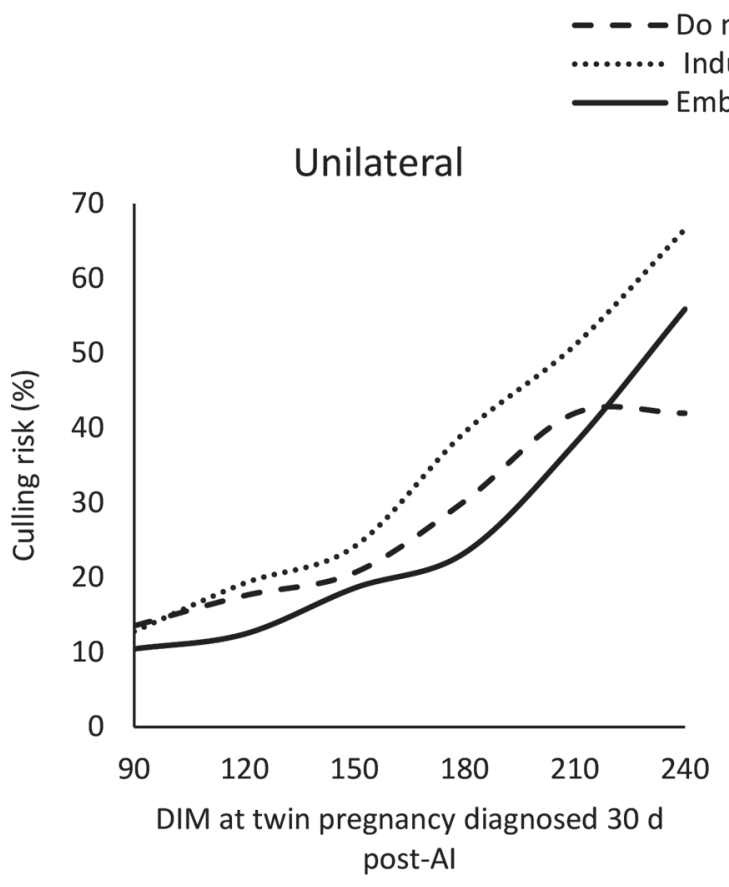

\section{Bilateral}

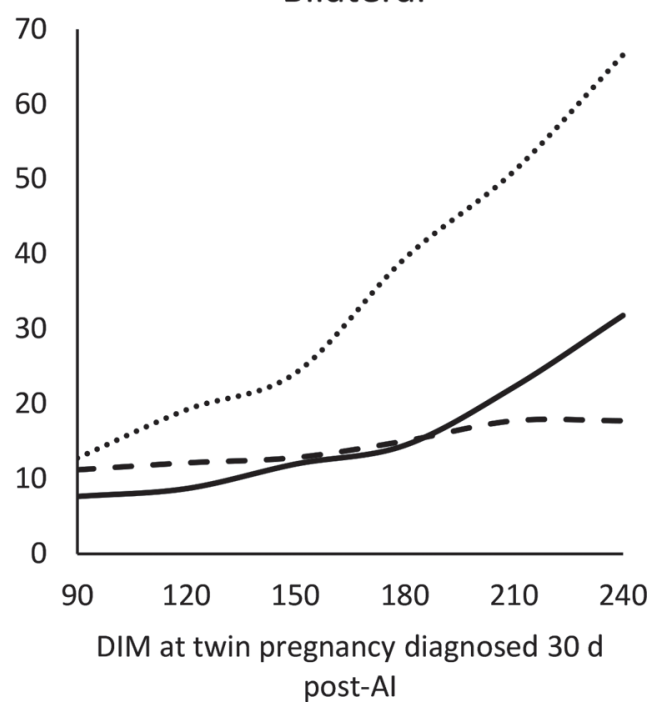

Figure 5. Culling risk due to reproductive reasons in the current lactation after different management options for a unilateral or bilateral twin pregnancy. 


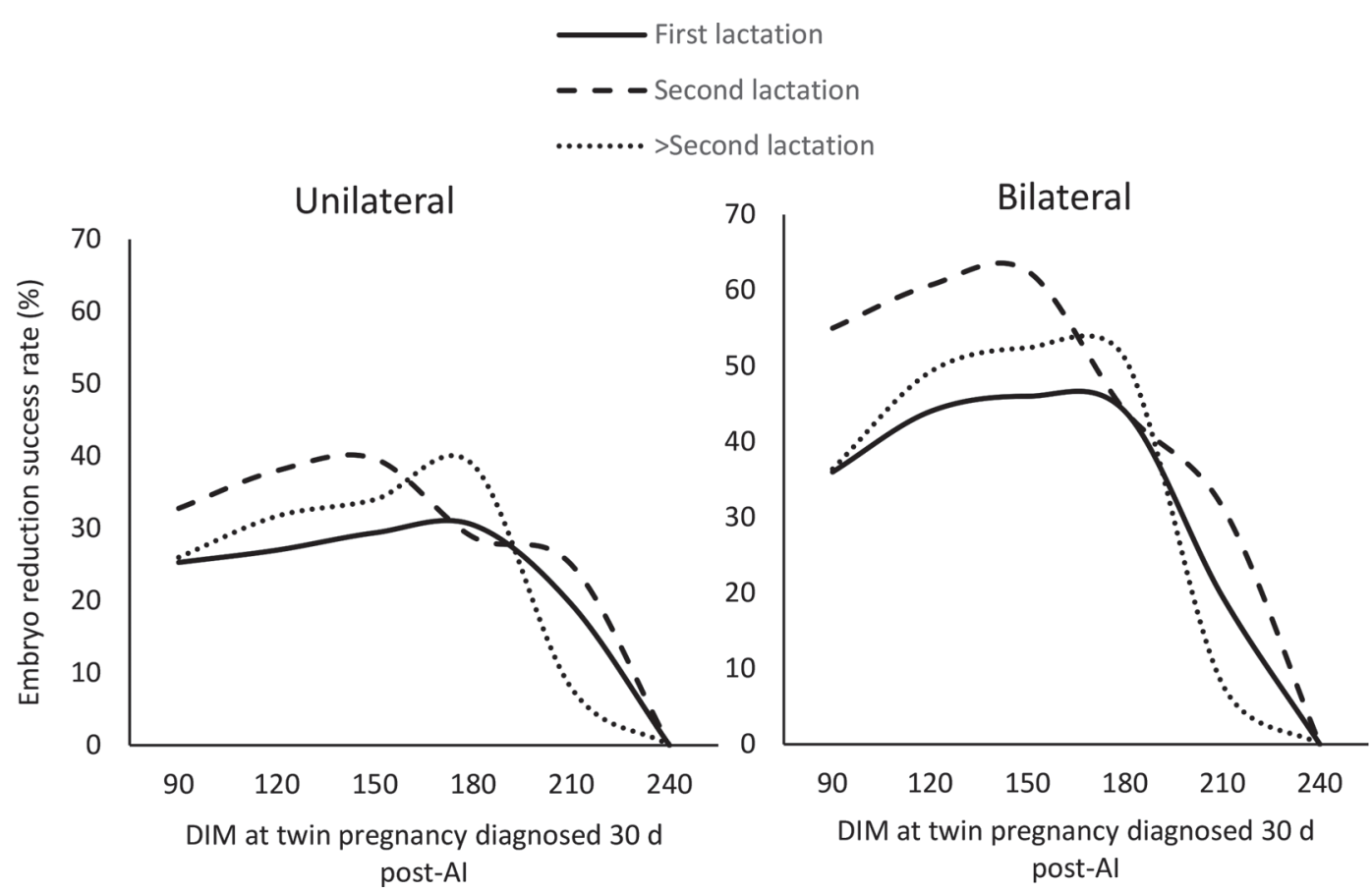

Figure 6. Minimum embryo reduction success rate for embryo reduction to remain the most economic management option after diagnosing a unilateral or bilateral twin pregnancy.

This specific scenario was chosen because it was the least-favorable scenario for manual embryo reduction. Under this scenario, manual embryo reduction presented the most similar cost when compared with the other options as well as the greatest breakeven point. If more scenarios are to be analyzed, a tool containing the model will be available at the University of WisconsinMadison Dairy Management website (http://dairymgt .info/tools.php).

In scenarios 2,3 , and 4 , the net value of the cows was increased. In scenario 2 , a cow yielding above farm average conceived twins; in scenario 3 , the herd average yield was increased; and, in scenario 4, the milk price was increased. As a result, in all those scenarios, the negative economic impact of a twin pregnancy was greater than in the baseline scenario, but the profit of carrying out manual embryo reduction with respect to the second-best option was greater. Thus, as the net value of a cow increased, the value of attempting manual embryo reduction increased, especially among the highest-yielding cows within a farm (Figure 7). In scenario 5 , the fertility after pregnancy loss or abortion was decreased. As expected, attempting manual embryo reduction decreased its net value with respect to the do nothing management option and a singleton pregnancy because it generated a higher culling rate.

Because not all cows diagnosed pregnant with twins would experience pregnancy loss at the average days in gestation reported in the literature (Table 1), scenario 6 introduced a twin pregnant cow undergoing pregnancy loss earlier during gestation. This scenario was designed to simulate the best possible scenario between all cows submitted to the do nothing management option. As forecasted, the net value of the do nothing management option was increased with respect to a single pregnancy and the manual embryo reduction management option, but even in this scenario, the manual embryo reduction management option remained the most economical alternative.

In some scenarios in Figure 7 (a), variations up to $55 \%$ in the net cost were related to variations lower than $3 \%$ in the breakeven point (Figure 7(b)). Thus, we can conclude that variations in the scenarios changed greatly the net cost of all management alternatives after diagnosing a twin pregnancy, but did not change significantly the relative ranking relationship between them. Thus, attempting manual embryo reduction remained the most economical option under a wide variety of scenarios.

\section{Model Advantages and Limitations}

As in any other simulation and modeling research, our study has some advantages as well as some limitations. The model was constructed to customize with easiness and detail the simulated farm, the specific cow 
Net profit of embryo reduction vs. do nothing (\$)

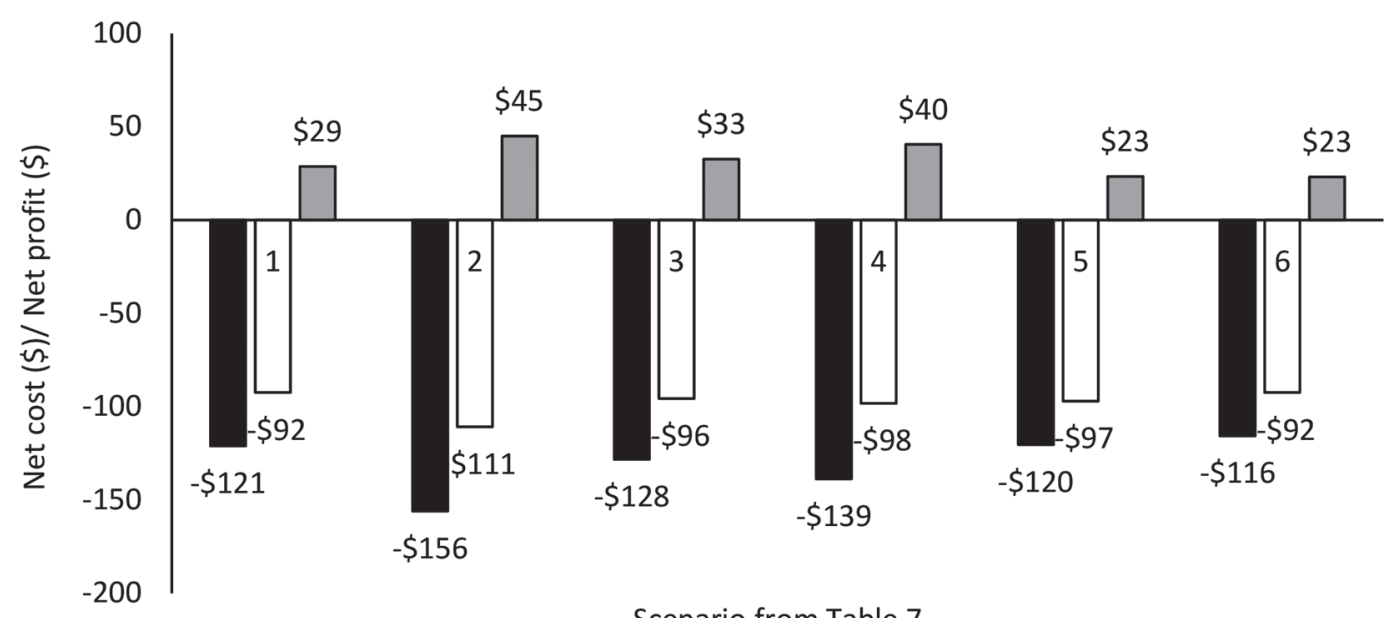

Scenario from Table 7

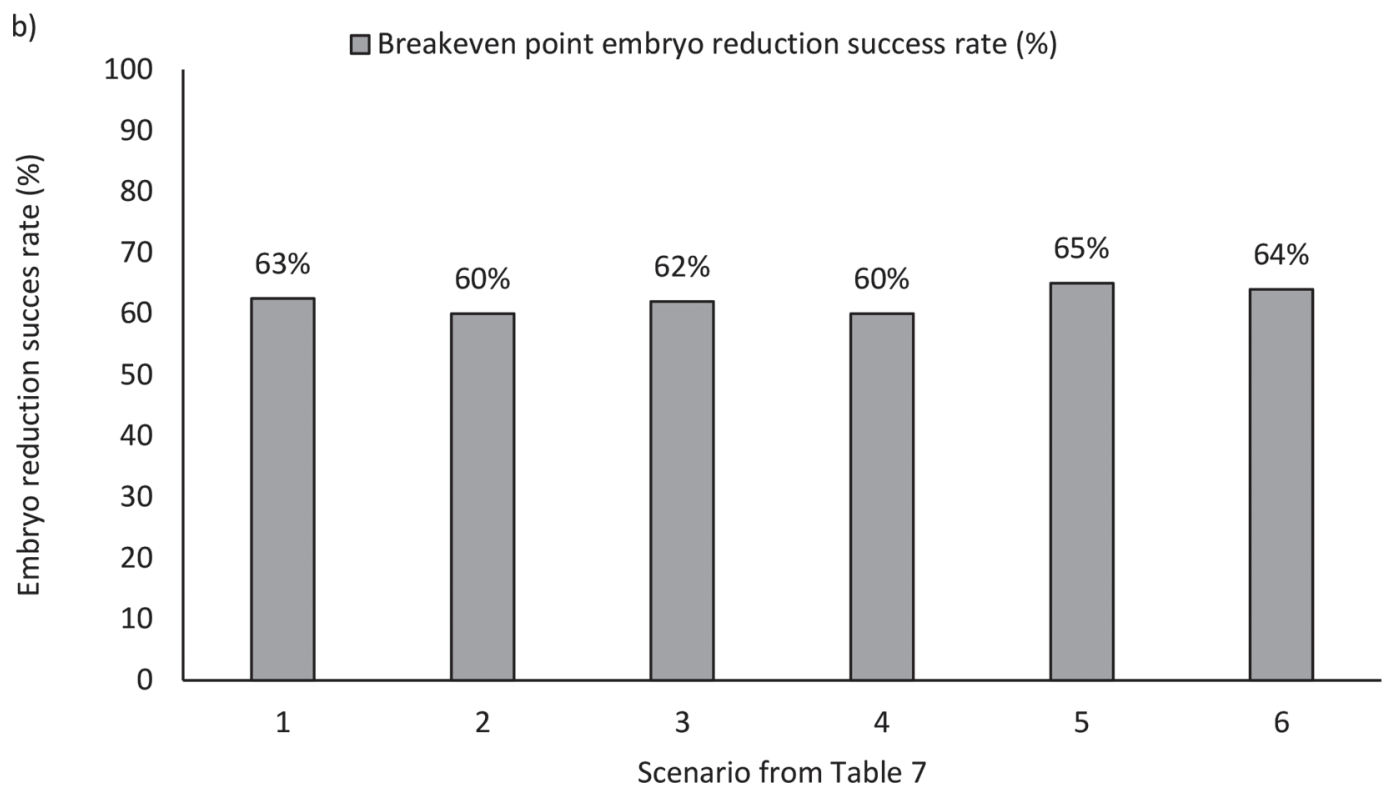

Figure 7. Net cost of different management strategies with respect to a single pregnancy, net profit of embryo reduction with respect to do nothing (a), and breakeven point for embryo reduction success (b) for the scenarios described in Table 7 for second-lactation cows diagnosed with a bilateral twin pregnancy at 150 DIM. 1 = baseline; $2=$ cow yielding $454 \mathrm{~kg}$ of milk per year more than average farm yield in baseline farm; $3=$ average cow in average farm with an average milk yield per cow per year $454 \mathrm{~kg}$ greater than the baseline farm; $4=$ baseline, with milk price increased $\$ 0.05$ per kg of milk; $5=$ baseline, with conception rate for first resynchronization after pregnancy loss and 21 -d pregnancy rate after resynchronization reduced $5 \% ; 6=$ baseline, with twin pregnancies losing gestation earlier, at the same time to single pregnancies.

diagnosed with twins, the market environment, and the effects of the different types of twin pregnancies. The model also allowed for an evaluation of the main effects of twinning (increased early pregnancy loss, increased abortion, increased retained placenta, increased metritis, and reduced lifespan) in the present and following lactations. The main limitations of the model were the (1) cows were immediately replaced at a minimum milk production threshold; (2) cows entering the simulation after a culling event behaved as the average cow on the farm without suffering pregnancy loss or culling until the end of the simulation period; (3) pregnancy loss, abortion, and early culling for cows diagnosed with twin pregnancies always occurred at the same day in gestation or lactation within every simulated management option; and (4) cows conceived twins only once during 
the simulation period. The first and second assumptions would have more negatively affected the management options with greater culling rate (i.e., the do nothing and induction of abortion management options; Figure 5). The third assumption was tested in the sensitivity analysis, which showed that even under the most favorable environment for the do nothing management option, the manual embryo reduction management option yielded the most economical outcome. The fourth limitation would have more negatively affected the induce abortion strategy because it was the option where more cows had to start a new gestation with the consequent risk of suffer twins again. In the remaining simulated lactations, the risk of conceiving twins again for cows that were not culled in the twin-simulated lactation would be higher than for new replacements coming into the simulation due to genetic predisposition for twinning. If recurrent twin gestation had been simulated, the overall cost of twinning would had increased for the 3 management options. Thus, although the model limitations could slightly change the absolute values obtained for the different management options, the ranking of relationships among them would not change, thereby maintaining the manual embryo reduction as the most economical management strategy to mitigate the negative economic impact of dairy cows diagnosed with twin pregnancies.

\section{CONCLUSIONS}

Twin pregnancies were estimated to have a negative effect of $\$ 96$ million per year on dairy farms in the United States. Management practices and genetic selection to decrease or prevent double ovulations and twin pregnancies in dairy cows are justified to be further developed. Moreover, twinning rate should be considered when economically evaluating different synchronization protocols or reproductive programs as well as in full-farm simulators. The results of the present study support that attempting embryo reduction for cows diagnosed with twins early during gestation was the most economical management strategy under a wide variety of scenarios evaluated. Methods should be studied and developed to improve the success rate for embryo reduction in dairy cows.

\section{ACKNOWLEDGMENTS}

Funding for Ramón Mur-Novales was provided through an FPI grant from the Spanish Ministry of Science and Innovation (MICINN, Madrid, Spain), BES-2013-063215.

\section{REFERENCES}

Andreu-Vázquez, C., I. Garcia-Ispierto, S. Ganau, P. M. Fricke, and F. López-Gatius. 2012a. Effects of twinning on the subsequent reproductive performance and productive lifespan of high-producing dairy cows. Theriogenology 78:2061-2070.

Andreu-Vázquez, C., I. Garcia-Ispierto, M. Lopez-Bejar, N. M. de Sousa, J. F. Beckers, and F. Lopez-Gatius. 2011. Clinical implications of induced twin reduction in dairy cattle. Theriogenology $76: 512-521$.

Andreu-Vázquez, C., I. Garcia-Ispierto, and F. López-Gatius. 2012b. Manual rupture versus transvaginal ultrasound-guided aspiration of allanto-amniotic fluid in multiple pregnancies: A clinical approach to embryo reduction in dairy cattle. J. Reprod. Dev. $58: 420-424$

Beerepoot, G. M., A. A. Dykhuizen, M. Nielen, and Y. H. Schukken. 1992. The economics of naturally occurring twinning in dairy cattle. J. Dairy Sci. 75:1044-1051.

Cabrera, V. E. 2012. A simple formulation and solution to the replacement problem: A practical tool to assess the economic cow value, the value of a new pregnancy, and the cost of a pregnancy loss. J. Dairy Sci. 95:4683-4698.

Cabrera, V. E. 2014. Economics of fertility in high-yielding dairy cows on confined TMR systems. Animal 8(Suppl. 1):211-221.

Eddy, R. G., O. Davies, and C. David. 1991. An economic assessment of twin births in British dairy herds. Vet. Rec. 129:526-529.

Ehrlich, J. L. 2011. Quantifying shape of lactation curves, and benchmark curves for common dairy breeds and parities. Bov. Pract. 45:88-95.

Fricke, P. M. 2001. Review: Twining in dairy cattle. Prof. Anim. Sci. 17:61-67.

Fricke, P. M. 2015. Double vision: Management of twinning in dairy cows. Proc. Am. Assoc. Bovine Pract. 48:116-124.

Giordano, J. O., A. S. Kalantari, P. M. Fricke, M. C. Wiltbank, and V. E. Cabrera. 2012. A daily herd Markov-chain model to study the reproductive and economic impact of reproductive programs combining timed artificial insemination and estrous detection. J. Dairy Sci. 95:5442-5460.

Johanson, J. M., P. J. Berger, B. W. Kirkpatrick, and M. R. Dentines. 2001. Twinning rates for North American Holstein sires. J. Dairy Sci. 84:2081-2088.

Kalantari, A. S., L. E. Armentano, R. D. Shaver, and V. E. Cabrera. 2016. Economic impact of nutritional grouping in dairy herds. J. Dairy Sci. 99:1672-1692.

Kinsel, M. L., W. E. Marsh, P. M. Ruegg, and W. G. Etherington. 1998. Risk factors for twinning in dairy cows. J. Dairy Sci. 81:989993.

Liang, D., M. Arnold, C. J. Stowe, R. J. Harmon, and J. M. Bewley. 2017. Estimating US dairy clinical disease costs with a stochastic simulation model. J. Dairy Sci. 100:1472-1486.

López-Gatius, F. 2005. The effect on pregnancy rate of progesterone administration after manual reduction of twin embryos in dairy cattle. J. Vet. Med. A Physiol. Pathol. Clin. Med. 52:199-201.

López-Gatius, F., C. Andreu-Vázquez, R. Mur-Novales, V. E. Cabrera, and R. H. F. Hunter. 2017. The dilemma of twin pregnancies in dairy cattle. A review of practical prospects. Livest. Sci. 197:12-16.

López-Gatius, F., and I. García-Ispierto. 2010. Ultrasound and endocrine findings that help to assess the risk of late embryo/early foetal loss by non-infectious cause in dairy cattle. Reprod. Domest. Anim. 45(Suppl 3):15-24.

López-Gatius, F., and R. H. F. Hunter. 2005. Spontaneous reduction of advanced twin embryos: its occurrence and clinical relevance in dairy cattle. Theriogenology 63:118-125.

López-Gatius, F., and R. H. F. Hunter. 2016. Twin reduction by $\mathrm{PGF}_{2 \alpha}$ intraluteal instillation in dairy cows. Reprod. Domest. Anim. 51:940-944.

López-Gatius, F., and R. H. F. Hunter. 2017. From pre-ovulatory follicle palpation to the challenge of twin pregnancies: Clinical reflections following on million gynaecological examinations in dairy cows. Reprod. Domest. Anim. 52(Suppl. 4):4-11. 
López-Gatius, F., P. Santolaria, J. L. Yaniz, J. M. Garbayo, and R. H. F. Hunter. 2004. Timing of early foetal loss for single and twin pregnancies in dairy cattle. Reprod. Domest. Anim. 39:429-433.

Markusfeld, O. 1987. Periparturient traits in seven high dairy herds. Incidence rates, association with parity, and interrelationships among traits. J. Dairy Sci. 70:158-166.

Nielen, M., Y. H. Schukken, D. T. Scholl, H. J. Wilbrink, and A. Brand. 1989. Twinning in dairy cattle: A study of risk factors and effects. Theriogenology 32:845-862.

NRC. 2001. Nutrient Requirements of Dairy Cattle. 7th ed. Natl. Acad. Sci., Washington, DC.

Padula, A. M. 2005. The freemartin syndrome: An update. Anim. Reprod. Sci. 87:93-109.

Pfeifer, L. F. M., A. Schneider, J. W. S. Neto, E. A. Ziguer, N. J. L. Dionello, and M. N. Corrêa. 2008. Biological and economic evaluation of flunixin meglumine in postpartum beef cows and heifers inseminated at fixed time. Rev. Bras. Zootec. 37:1392-1397.

Santos, V. G., P. D. Carvalho, C. Maia, B. Carneiro, A. Valenza, P. M. Crump, and P. M. Fricke. 2016. Adding a second prostaglandin $\mathrm{F}_{2 \alpha}$ treatment to but not reducing the duration of a PRID-Synch protocol increases fertility after resynchronization of ovulation in lactating Holstein cows. J. Dairy Sci. 99:3869-3879.
Silva del Río, N., J. Colloton, and P. M. Fricke. 2009. Factors affecting pregnancy loss for single and twin pregnancies in a high-producing dairy herd. Theriogenology 71:1462-1471.

Silva del Río, N., P. M. Fricke, and R. R. Grummer. 2010. Effects of twin pregnancy and dry period feeding strategy on milk production, energy balance and metabolic profiles in Holstein cows. J. Anim. Sci. 88:1048-1060.

Silva del Río, N., S. Stewart, P. Rapnicki, Y. M. Chang, and P. M. Fricke. 2007. An observational analysis of twin births, calf sex ratio, and calf mortality in Holstein dairy cattle. J. Dairy Sci. 90:1255-1264.

USDA. 2017. United States Department of Agriculture. Milk Production Report. USDA, Washington, DC. http://usda.mannlib.cornell .edu/MannUsda/viewDocumentInfo.do?documentID=1103.

van Arendonk, J. A. M. 1985. A model to estimate the performance, revenues and costs of dairy cows under different production and price situations. Agric. Syst. 16:157-189.

Wiltbank, M. C., P. M. Fricke, S. Sangritasvong, R. Sartori, and O. J. Ginther. 2000. Mechanisms that prevent and produce double ovulations in dairy cattle. J. Dairy Sci. 83:2998-3007. 